\title{
Comparative study between the effect of stem cells and placental extract on streptozotocin damaged liver in diabetic rats
}

\author{
Nehal M. Eisa ${ }^{1}$, Shereen S. Elshaer ${ }^{1,2}$ and Noha A. Eldesoky ${ }^{1 *}$ \\ ${ }^{1}$ Department of Biochemistry and Molecular Biology, Faculty of Pharmacy (Girls), Al-Azhar University, Cairo, Egypt. \\ ${ }^{2}$ Department of Biochemistry, Faculty of Pharmacy, Heliopolis University for Sustainable Development, Cairo, Egypt. \\ * Correspondence: noha-bio@azhar.edu.eg (ORCID ID: 0000-0002-0680-454x)
}

Article history: Received 2021-04-01 Revised 2021-06-24

Accepted 2021-07-11

\begin{abstract}
Streptozotocin (STZ) is a pancreatic beta cells specific cytotoxin that causes direct organ toxicity and diabetes. Organ toxicity caused by STZ can affect variety of organs, including the liver. The present study discusses the impact of mesenchymal stem cells (MSCs) and placental extract (PE) on the streptozotocin damaged livers in rats. The animals in this study were dichotomized into five groups, each with ten rats: group I (negative controls) and the other forty rats were given $50 \mathrm{mg} / \mathrm{kg} \mathrm{STZ} \mathrm{for} \mathrm{diabetes} \mathrm{induction} \mathrm{and} \mathrm{then} \mathrm{divided}$ into; group II (positive controls), group III (MSCs treated), group IV (PE treated), and group V (MSCs/PE combination treated). Animals were slaughtered after four weeks of therapy, and serum was isolated for ALT, AST, and ALP determination. For histopathological analysis, liver tissues were sliced, washed in normal saline, and fixed in $10 \%$ formalin. When compared to positive controls, the three treated groups showed a major improvement in liver function parameters $(P<0.05)$. Histopathological examination showed great improvement in the three treated groups where group $\mathrm{V}$ revealed the best picture. The present study points to the possible effects of PE and MSCs in the treatment of streptozotocin damaged liver in diabetic rats. This action could be ascribed to MSCs homing in the liver and the restoration of liver tissues by PE contents of growth factors. These actions give a hope for diabetic patients suffering from variety of complications since the regeneration effects of MSCs and PE have been expanded to include other damaged tissues.
\end{abstract}

Keywords: Mesenchymal stem cells (MSCs); Streptozotocin (STZ); placental extract (PE); Liver diseases; Diabetes.

This is an open access article distributed under the CC BY-NC-ND license https://creativecommons.org/licenses/by/4.0/

\section{INTRODUCTION}

Diabetes mellitus is a common health condition with multiple etiologies that is primarily characterized by hyperglycemia caused by insulin secretion and/or function abnormalities. It's also linked to liver complications, including excessive liver enzymes, fatty liver disease, cirrhosis, hepatocellular carcinoma, and acute liver failure ${ }^{\mathbf{1 , 2}}$. Chronic liver disease has become more common in the last decade as a result of metabolic syndrome, which includes abdominal obesity and insulin resistance ${ }^{3}$. Insulin resistance is a main cause of type 2 diabetes mellitus, as it contributes to hyperglycemia and oxidative stress, which causes liver tissue necrosis ${ }^{4}$.

The liver is primarily responsible for maintaining glucose homeostasis. Numerous studies have explained that oxidative stress induces diabetes mellitus by causing the formation of reactive oxygen species (ROS), which are the main cause of cellular harm ${ }^{4-6}$. As a result, diabetes mellitus is strongly linked to liver inflammation, cirrhosis, apoptosis, and -cell dysfunction, that contribute to liver dysfunction ${ }^{7}$.

Streptozotocin (STZ), which has been shown to be harmful to pancreatic islet beta cells, has been used to cause diabetes mellitus in many animal species, and the results usually resemble human hyperglycemic non-ketotic DM ${ }^{8}$. In experimental animals, morphological and functional changes in various body organs, including the liver, that accompany STZ-induced diabetes are useful to be studied $\mathbf{9 , 1 0}$.

In regenerative medicine, mesenchymal stem cells (MSCs) have been outcropped as a therapeutic technique. MSCs can differentiate into various cell types from all three germ layers, as well as migrate to damaged sites and exert their distinctive

Cite this article: Eisa, N., Eldesoky, N., Elshaer, S. Comparative Study between the Effect of Stem Cells and Placental Extract on Streptozotocin Damaged Liver in Diabetic Rats. Azhar International Journal of Pharmaceutical and Medical Sciences, 2022; 2 (1): 58 -65. doi: 10.21608/aijpms.2021.210566 
immunomodulatory actions, so they became attractive therapeutic tools for tissue engineering and tissue/organ injury repair 11. Approximately, 321 MSC-based clinical trials have been completed. Nearly half of all MSC-based clinical trials focused on bone/cartilage, brain/Nero, and immune-related diseases. In addition, there are 16 clinical trials involving liver diseases. On the other hand, tissue damage and over-activation of inflammatory pathways are also present in liver diseases. As a result, MSCs could be the best candidate for cell therapy of liver diseases ${ }^{\mathbf{1 2}}$.

The placenta contains the genetic material required to generate all cell types and is very rich in nutrients ${ }^{13}$. Placental extract has been commonly used in traditional medicine for wound healing and as an anti-inflammatory agent. People from many countries used placental extract as a drug of renovation and vitalization ${ }^{\mathbf{1 4}}$.

Placental extracts have a wide range of therapeutic effects, including tissue regeneration, wound healing, immunomodulation, antiinflammation, and cellular proliferation $\mathbf{1 5}$. Furthermore, for more than 40 years, human placental extract has been used in traditional folk medicine, especially in China, to treat liver diseases such as chronic hepatitis and liver cirrhosis ${ }^{\mathbf{1 6}}$. It had the ability to promote liver regeneration while also inhibiting inflammatory reactions and hepatocyte apoptosis. Hepatocyte growth factor is one of its main active ingredients ${ }^{\mathbf{1 4}}$. Human placental extract is currently permitted for clinical use in several countries to treat chronic liver diseases and menopausal symptoms ${ }^{17}$.

Placental extract is manufactured by the hydrolyzation of placental cells. The final extract contains collagen, elastin, laminin, trace elements, peptides, cytokines and Polydeoxyribonucleotide (PRDN) ${ }^{18}$. PE also includes essential and nonessential amino acids, which in vitro promote fibroblast proliferation and collagen formation ${ }^{\mathbf{1 9}}$. High concentrations of vitamins that promote energy production and cellular metabolism, as well as high concentrations of growth factors with antiinflammatory and reformative properties, are found in placental extracts ${ }^{\mathbf{1 8}}$.

The current research is a part of a study done on STZ-induced diabetic rats. In the present part we discuss the therapeutic effects of MSCs and PE alone or in combination on damaged livers of STZ-induced diabetic rats to determine the best therapeutic regimen.

\section{METHODS}

\subsection{Chemicals:}

\subsubsection{Streptozotocin:}

Obtained from (Sigma Chemical Company) ( $\mathrm{St}$ Louis, Missouri, USA) in the form of lyophilized powder, freshly disbanded in $0.1 \mathrm{M}$ citrate buffer $(\mathrm{pH}$ $=4.5)$ used at a dose of $(50 \mathrm{mg} / \mathrm{kg}$ body weight $)$ within 15 minutes of dissolution ${ }^{\mathbf{2 0}}$.

\subsubsection{Mesenchymal stem cells:}

$20 \times 10^{6}$ cells in Dulbecco's modified Eagles medium (DMEM) were prepared in tissue culture laboratory at Genetic Engineering Centre (Al-Azhar University) according to the method of Abdel Aziz et al. ${ }^{21}$ and given in two doses via intravenous injection into the tail vein:

1. First dose $\left(10^{6}\right.$ cells/ rat $)$ in $0.2 \mathrm{ml}$ DMEM.

2. After a week, the rats were given the 2 nd dose of cells $\left(10^{6}\right.$ cells/ rat) in $0.2 \mathrm{ml}$ DMEM.

\subsubsection{Placental extract:}

Laennec $₫$ is a human placental extract (Japan Bio Products Co., LTD, Tokyo, Japan). PE was given intravenously via the tail vein on alternating days for four weeks at a dosage of $40 \mu 1 /$ rat $^{\mathbf{2 2}}$, as directed by the manufacturer.

\subsection{Experimental design:}

Adult male albino rats weighing $180 \pm 20 \mathrm{gm}$ were obtained from Al-Nile Company of Pharmaceutical Products (Cairo, Egypt). They were nourished regular pellet chow (El-Nasr Chemical Company, Cairo, Egypt) and allowed to free access to water. For acclimatization, the animals were kept in the same circumstances for a week before the experiment.

The present study included fifty animals which were divided into five groups:

Group (1): negative control group (vehicle only was given to normal rats).

For induction of diabetes ${ }^{\mathbf{2 0}}$, the four other groups received a single dose of Streptozotocin (50 $\mathrm{mg} / \mathrm{kg}$ body weight) dissolved in $0.2 \mathrm{~mL}$ citrate buffer ( $\mathrm{pH} 4.5$ ). After 72 hours of STZ dose, diabetes was verified by a random blood glucose level of $>300$ $\mathrm{mg} / \mathrm{dl}^{23}$.

The other four groups are:

Group (2): Diabetic rats that have not been treated (positive control group).

Group (3): Diabetic rats treated with MSCs; injected twice $\left(10^{6}\right.$ cells/ rat) in $0.2 \mathrm{ml}$ DMEM with a break one week between both doses ${ }^{24}$.

Group (4): Diabetic rats treated with placental extract $(40 \mu \mathrm{l} / \mathrm{rat}$ of $\mathrm{PE})$ on alternating days for 4 weeks. 
Group (5): Diabetic rats received a combination of MSCs and PE by the same dosage protocol (MSCs: $10^{6}$ cells/ rat) and (PE: $40 \mu 1 /$ rat).

Animals were sacrificed after four weeks of treatment; livers were sliced, washed in regular saline, and fixed in $10 \%$ formalin.

\subsection{Biochemical tests included:}

Serum alanine aminotransferase (ALT), aspartate aminotransferase (AST) and alkaline phosphatase (ALP) were determined by spectrophotometric technique according to the manufacturer's instructions ${ }^{25}$.

\subsection{Statistical methods:}

For data analysis, IBM SPSS statistics (V. 26.0, IBM Corp., USA, 2019) was used. Data were expressed as median and percentiles for quantitative non-parametric measures. Wilcoxon Rank Sum test was used for comparison between two independent groups. The Kruskal-Wallis test was used to compare the outcomes of more than two groups. The probability of error $<0.05$ was considered significant, while $<0.01$ was highly significant.

\section{RESULTS}

3.1 Table 1. Median ( $1^{\text {st }}$ quartile $-3^{\text {rd }}$ quartile) of liver function tests in all studied groups.

\begin{tabular}{cccccc}
\hline Parameter & $\begin{array}{c}\text { Gp.1 } \\
\text { no=10 }\end{array}$ & $\begin{array}{c}\text { Gp.2 } \\
\text { no=10 }\end{array}$ & $\begin{array}{c}\text { Gp.3 } \\
\text { no=10 }\end{array}$ & $\begin{array}{c}\text { Gp.4 } \\
\text { no=10 }\end{array}$ & $\begin{array}{c}\text { Gp.5 } \\
\text { no= } \mathbf{1 0}\end{array}$ \\
\hline ALT & 60.5 & $120.1^{\mathrm{a}}$ & $69.2^{\mathrm{b}}$ & $67.2^{\mathrm{b}}$ & $62.6^{\mathrm{b}}$ \\
(U/L) & $(55.6-67.3)$ & $(105.2-131.5)$ & $(60-76.8)$ & $(62-74.7)$ & $(56.9-68.5)$ \\
\hline AST & 44.2 & $80.3^{\mathrm{a}}$ & $45^{\mathrm{b}}$ & $45^{\mathrm{b}}$ & $44.4^{\mathrm{b}}$ \\
(U/L) & $(42-46.4)$ & $(76.1-83.9)$ & $(46.1-51.4)$ & $(46.2-51.1)$ & $(45.9-49.4)$ \\
\hline ALP & 44.1 & $190.5^{\mathrm{a}}$ & $53.3^{\mathrm{b}}$ & $50.1^{\mathrm{b}}$ & $45.5^{\mathrm{b}}$ \\
(U/L) & $(42-46.4)$ & $(165-240)$ & $(50-58.5)$ & $(46-55.5)$ & $(43-47.8)$ \\
\hline
\end{tabular}

${ }^{\text {a }}$ Significant from group $1,{ }^{\mathrm{b}}$ Significant from group 2 (at $P$ value $\leq 0.05$ ).

\subsection{Histopathological Investigations:}

\subsubsection{Negative control group:}

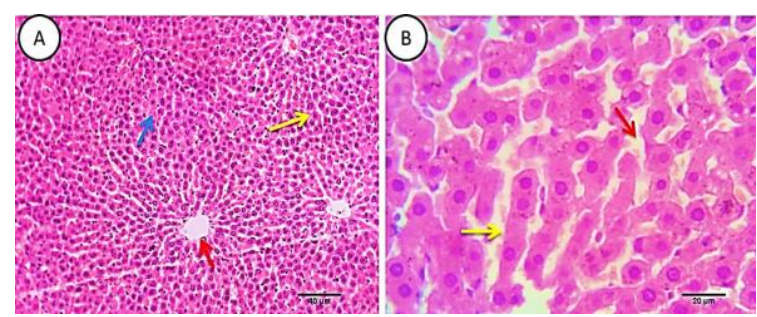

Figure 1. Photomicrograph of liver of group 1 which shows typical hepatic parenchyma with preserved lobular pattern (blue arrow), central veins (A, red arrow), sinusoids (B, red arrow) and hepatic cords arrangement. (yellow arrows). Scale bars 40, 20 um.

\subsubsection{Positive control group:}

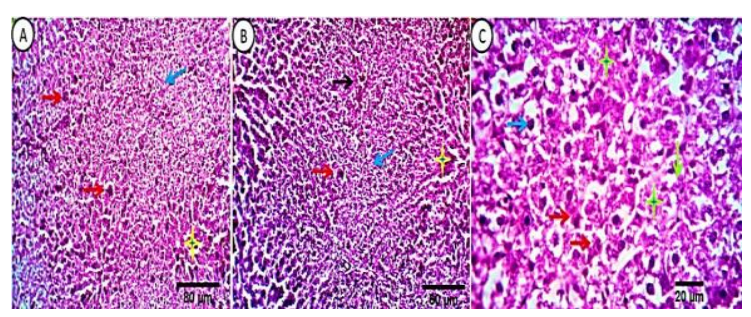

Figure 2. Photomicrograph of liver of group 2 shows marked multifocal peri-lobular hepatocyte degenerative and necrotic changes (blue arrows, green arrows and stars), apoptosis of variable number of hepatocytes (red arrows) and vascular dilatation with erythrocytic enlargement in the surrounding blood vessel and sinusoids (yellow stars). Scale bars 80, 60, 20 um.

\subsubsection{MSCs treated group:}

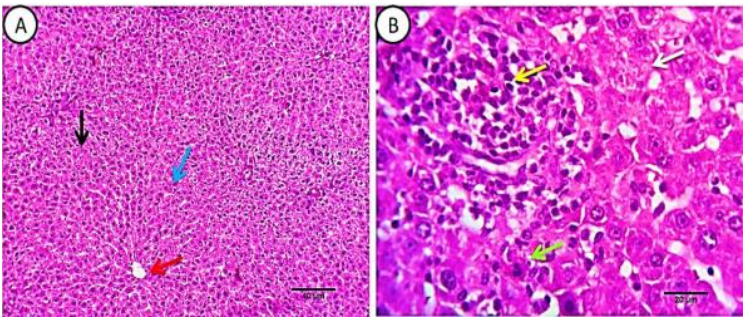

Figure 3. Photomicrograph of liver of MSCs- treated diabetic rats showing:

(A) apparently normal structures with preserved lobular pattern (blue arrow), hepatic cords (black arrow) and vascular arrangement (red arrow) apart of the few degenerated hepatocytes (hydropic degeneration) (white arrow).

(B) hepatocellular necrotic areas, replaced by round cells) (lymphocytes and plasma cells (yellow arrow), few hepatocytes show degenerative and apoptotic changes (white and green arrows). Scale bars 40, 20 um. 


\subsubsection{Placental extract treated group:}

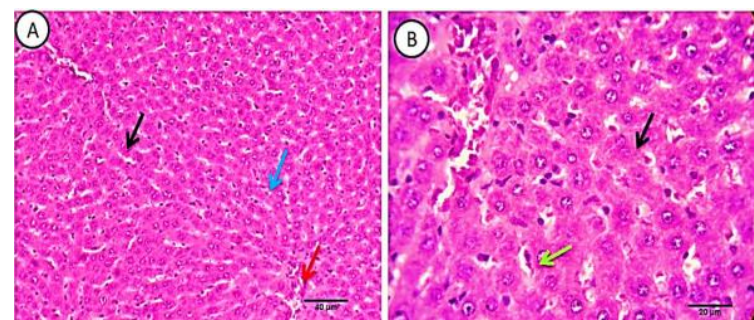

Figure 4. Photomicrograph of liver of PE-treated diabetic rats showing apparently normal histo-morphological features of hepatic parenchyma and stroma with almost normal hepatocytes (blue arrow), cord arrangement (black arrows) and vascular structures (red and green arrows). Scale bars 40, 20 um.

\subsubsection{MSCs/PE Combination treated group:}

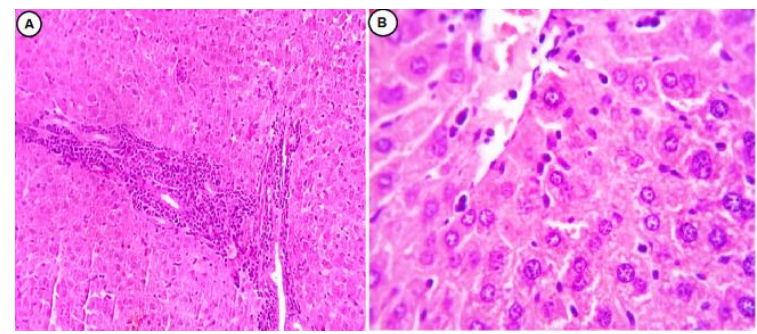

Figure 5. Photomicrograph of liver of combination treated group showing apparently normal hepatic parenchyma with regard to hepatocytes, central vein, sinusoids and Von Kupffer cells which appears slightly hypertrophied. Scale bars 40, 20 um.

\section{DISCUSSION}

Diabetes-related hepatotoxicity has received less attention than other common complications such as cardiopathy, retinopathy, and nephropathy ${ }^{1,26}$.

The aim of this research was to explore how MSCs and placental extract could affect the damaged livers of STZ-induced diabetic rats. Elevated serum aminotransferases (AST, ALT), and ALP activities are typical manifestations of liver disease, and have been found to be more affected in diabetic patients than in the general population ${ }^{9}$.

Previous human and animal studies used levels of ALT, AST, and ALP to determine the hepatic effects of DM ${ }^{27}$. The present study showed that liver enzymes (ALT, AST and ALP) levels were significantly increased in group 2 than group 1 . This effect was similar to the results of other studies that found STZ detrimental to liver function ${ }^{1}$.

The elevated aminotransferases level was explained by the cellular damage in the liver caused by STZ induction. While ALT can be found in both mitochondria and cytosol, the mitochondrial form has a low activity and is highly unstable; hence almost all detected ALT is cytosolic in origin. However, the precise mechanism by which enzymes are emitted from the cytosol and mitochondria of hepatocytes is not well understood. Experiments have shown that minor membrane changes are necessary to enable intracellular enzymes to migrate into the extracellular space. The presence of very large concentration gradient between the hepatocytes and the sinusoidal space usually exists for enzymes also play a role. Besides, cell damage increases permeability causing cytosolic isoenzymes to spill into the sinusoids and from there into the peripheral blood ${ }^{9}$.

Rodríguez et al. ${ }^{28}$ mentioned that STZ in hyperglycemic animals causes a time dependent rise in AST, ALT, and ALP levels. Also, it has been shown that STZ produces alterations in hepatic functions in poorly controlled diabetics ${ }^{2}$. Other studies have reported hepatocellular dysfunction after 1-3 weeks of diabetes induction ${ }^{9}$. The changes in hepatic function may be attributed to a rise in araginase activity and mRNA levels of aminotransferases ${ }^{29}$.

In the current study, histopathological examination of liver tissues of positive control group revealed a toxic hepato-cellular destructive effect of the injected STZ compound, as evidenced by multifocal peri-lobular hepatocyte degenerative and necrotic changes, apoptosis of a variable number of hepatocytes, and vascular dilatation with erythrocytic engorgement in the surrounding blood vessel and sinusoids. In certain portal regions, there was a minor lymphoplasmacytic reaction. The biliary system was the least affected, with only few bile ducts and ductules showing proliferative changes (Fig. 2). These outcomes were in line with Al-Ani et al. ${ }^{30}$ who discovered massive pathological changes in the livers of STZ diabetic rats as compared to the usual structure observed in the negative control.

In the present study, MSCs had positive effects on the liver tissues of STZ diabetic rats appeared as reduction of liver enzymes (table 1). Furthermore, histopathological examination of MSCs treated group was apparently normal with preserved lobular pattern, hepatic cords and vascular structure, as well as few degenerated and apoptotic hepatocytes (Fig. 3 ). These results were in harmony with the results of Yang et al. who reported that MSC transplantation could promote partial recovery of liver function and alleviate liver inflammation in several animal models of liver fibrosis or cirrhosis by direct and indirect effects of MSCs on the fate of activated hepatic 
stellate cells, differentiation of MSCs into Hepatocyte-like cells and immunomodulation capacity of MSCs ${ }^{12}$.

Khalil et al. demonstrated that MSCs transplantation reduced serum AST and ALT, restored liver construction and activity in a rat model of $\mathrm{CCL}_{4}$-induced liver fibrosis, reducing $\mathrm{CCl}_{4}$ toxicity and enhancing liver function ${ }^{31}$. Also, Zheng et al. ${ }^{32}$, Huang et al. ${ }^{33}$ and Gilsanz et al. ${ }^{34}$ found that transplantation of MSC can enhance function of liver, hinder hepatocyte apoptosis, and stimulate proliferation of hepatocyte in animal models of acute liver failure. Moreover, Zhang et al. ${ }^{35}$ found that transplantation of MSC reduced liver enzymes levels when compared with an acute liver damage model. Furthermore, MSC transplantation in rats could control metabolic disorders in the liver and blood, such as an excess of amino acids, bile acids, sphingolipids, acylcarnitines, and glycerophospholipids which increase proliferation and decrease hepatocytes apoptosis ${ }^{36}$.

In the current study, placental extract treated group had significantly lower levels of liver enzymes when contrasted to the positive control group (table 1). Also, Histopathological examination showed evidence of cellular regeneration, with typical histomorphological features of hepatic parenchyma and stroma, as well as normal hepatocytes, cord organization, and vascular structures in the majority of the examined sections of this group. (Fig. 4). These promising results reflect a great improvement in the liver condition and make us recommend its trial in humans with liver diseases for many reasons; First: PE is a mixture of some proteins, growth factors, vitamins and trace elements so it could be administered safely especially it has been already used in many other diseases with almost no reported side effects. ${ }^{18}$ Second: some researchers reported promising actions of PE against immune-mediated hepatitis ${ }^{14}$. Third: placental extract injection was reported to have anti-inflammatory and regenerative effects on damaged tissues that could help with various liver injuries ${ }^{19}$.

Similar results were observed in $\mathrm{MSCs} / \mathrm{PE}$ combination treated group, in which liver enzymes returned to their normal levels. Furthermore, histopathological examination of combination treated group showed apparently normal hepatic parenchyma with moderate portal aggregation of mono-nuclear cells (lymphocytes and macrophages) and mild biliary proliferation (Fig. 5). These results came in line with our anticipations due to the collection of benefits of both MSCs and PE.

Naughton et al. ${ }^{37}$ and Yamauchi et al. ${ }^{38}$ stated that placental extract has been used for thousands of years in China and in Korea as a therapeutic agent for liver renewal especially in traditional folk medicine.
Liu et al. found that placental extract of sheep had possible therapeutic effects against immunemediated hepatitis ${ }^{14}$ and Landek et al. reported that placental proteins greatly reduced over all serum levels of $\mathrm{IgG}$ and $\operatorname{IgM}$ thyroglobulin-specific antibodies in experimental autoimmune thyroiditis 39 .

All of the treated groups in this study (MSCs treated, PE treated and MSCs/PE combination treated groups) showed great improvements in liver functions, with liver enzymes returning to normal levels (table 1). Moreover, no significant differences between all these groups regarding ALT, AST and ALP levels were observed reflecting the efficacy of all the used regimens in the treatment of liver affection related to diabetes.

\section{CONCLUSIONS}

This study reported important roles of MSCs and placental extract either alone or in combination regarding the regeneration and proliferation of liver tissue in streptozotocin induced diabetic rats, as evidenced by the improved liver enzymes and histopathological examination of the liver tissues.

Funding: This research did not receive any specific grant from funding agencies in the public, commercial, or not-for-profit sectors.

Acknowledgments: We'd like to express our deep gratitude to Tissue Culture and Molecular Biology team, Genetic Engineering Center, Al-Azhar University and to the animal house and histopathology teams, Faculty of Science, Al-Azhar University for their great efforts during the practical part of this work.

Conflicts of Interest: The authors declare no conflict of interest.

Ethical Statement: Animal in-vivo procedures involved in the present study was carried out in conjunction with the National Institutes of Health's guidelines for the care and use of Laboratory animals (NIH Publications No. 8023, revised 1978) ${ }^{40}$. The "Research Ethics Committee" for "Animal Care and Use" Faculty of Pharmacy (Girls), Al-Azhar University, Cairo, Egypt (REC no.158) reviewed and accepted the research protocol.

Author Contribution: Nehal Mohamed Eisa: Investigation, Practical Work, Formal analysis and Writing Original Draft \& Review, Resources and Software. Shereen Saeid Elshaer: Validation of Data, Methodology, Final Manuscript Revision and Supervision. Noha Abdel-Rahman Eldesoky: Conceptualization, Data Visualization and Curation, Editing manuscript, Management and Coordination. 
List of Abbreviations:

MSCs: Mesenchymal stem cells, PE: Placental extract, STZ: Streptozotocin, DM: Diabetes mellitus, ROS: Reactive oxygen species, PRDN: Polydeoxyribonucleotide, DMEM: Dulbecco's modified Eagles medium, ALT: Alanine aminotransferase, AST: Aspartate aminotransferase, ALP: Alkaline phosphatase.

\section{REFERENCES}

1. El Gawly HW, Tawfik MK, Rashwan ME, Baruzaig AS. The effect of pioglitazone on the liver of streptozotocin-induced diabetic albino Wistar rats. Eur Rev Med Pharm Sci. 2009; 13:43-451.

2. Yazdi HB, Hojati V, Shiravi A, Hosseinian S, Vaezi G, Hadjzadeh MAR. Liver dysfunction and oxidative stress in streptozotocin-induced diabetic rats: protective role of Artemisia turanica. Journal of pharmacopuncture. 2019; 22(2): 109. doi: 10.3831/KPI.2019.22.014.

3. Kohl T, Gehrke N, Schad A. Diabetic liver injury from streptozotocin is regulated through the caspase- 8 homolog cFLIP involving activation of JNK2 and intrahepatic immunocompetent cells. Cell Death \& Disease. 2013; 4 (7): 712. doi: 10.1038/cddis.2013.228.

4. Ghosh S, Bhattacharyya S, Rashid K, Sil PC. Curcumin protects rat liver from streptozotocin-induced diabetic pathophysiology by counteracting reactive oxygen species and inhibiting the activation of p53 and MAPKs mediated stress response pathways. Toxicology Reports. 2015; 2: 365-376. doi: 10.1016/j.toxrep.2014.12.017.

5. Chattopadhyay M, Khemka VK, Chatterjee G, Ganguly A, Mukhopadhyay S, Chakrabarti S. Enhanced ROS production and oxidative damage in subcutaneous white adipose tissue mitochondria in obese and type 2 diabetes subjects. Mol Cell Biochem 2015; 399: 95-103.

6. He L, He T, Farrar S, Ji L, Liu T, Ma $\mathrm{X}$. Antioxidants maintain cellular redox homeostasis by elimination of reactive oxygen species. Cellular Physiology and Biochemistry. 2017; 44 (2): 532-553.

7. Safhi MM, Alam MF, Sivakumar SM, Anwer T. Hepatoprotective potential of
Sargassum muticum against STZ-induced diabetic liver damage in wistar rats by inhibiting cytokines and the apoptosis pathway. Analytical Cellular Pathology. 2019; 2019: 7958701.doi: 10.1155/2019/7958701.

8. Wu J, Yan LJ. Streptozotocin-induced type 1 diabetes in rodents as a model for studying mitochondrial mechanisms of diabetic $\beta$ cell glucotoxicity. Diabetes, metabolic syndrome and obesity: targets and therapy. 2015; 8: 181. doi: 10.2147/DMSO.S82272.

9. Salih ND, Kumar GH, Noah RM, Muslih RK. The effect of streptozotocin induced diabetes mellitus on liver activity in mice. Global Journal on Advances Pure and Applied Sciences. 2014; 4.

10. Lucchesi AN, Cassettari LL, Spadella CT. Alloxan-induced diabetes causes morphological and ultrastructural changes in rat liver that resemble the natural history of chronic fatty liver disease in humans. Journal of diabetes research. 2015; 2015. doi:10.1155/2015/494578.

11. Zakrzewski W, Dobrzyński M, Szymonowicz M, Rybak Z. Stem cells: past, present, and future. Stem cell research \& therapy. 2019; 10(1):68. doi:10.1186/s13287-019-1165-5.

12. Yang X, Meng Y, Han Z, Ye F, Wei L, Zong C. Mesenchymal stem cell therapy for liver disease: full of chances and challenges. Cell \& Bioscience. 2020; 10(1): 1-18. doi: 10.1186/s13578-02000480-6.

13. Pogozhykh O, Prokopyuk V, Figueiredo C, Pogozhykh D. Placenta and placental derivatives in regenerative therapies: experimental studies, history, and prospects. Stem Cells International. 2018; doi:10.1155/2018/4837930.

14. Liu J, Luo S, Yang J, Ren F, Zhao Y, Luo $\mathrm{H}$, et al. The protective effect of sheep placental extract on concanavalin Ainduced liver injury in mice. Molecules. 2019; 24(1): 28. doi:10.3390/molecules24010028.

15. Pan SY, Chan MK, Wong MB, Klokol D, Chernykh V. Placental Therapy: An insight to their biological and therapeutic properties. Blood. 2017; 4(11): 12.

16. Yamauchi A, Kamiyoshi A, Koyama T, Iinuma N, Yamaguchi S, Miyazaki H, et al. Placental extract ameliorates nonalcoholic steatohepatitis (NASH) by 
exerting protective effects on endothelial cells. Heliyon. 2017; 3(9): e00416.doi: 10.1016/j.heliyon.2017.e00416.

17. Azayeri MH, Nedaeinia R, Aghaie T, Motallebnezhad M. Human placental extract attenuates neurological symptoms in the experimental autoimmune encephalomyelitis model of multiple sclerosis-a putative approach in MS disease. Autoimmunity Highlights. 2020; 11(1): 1-9. doi: 10.1186/s13317020-00137-x.

18. Gwam C, Ohanele C, Hamby J. Human placental extract: a potential therapeutic in treating osteoarthritis. Ann Transl Med. 2020; doi: 10.21037/atm. 2019.10.20.

19. Park KM, Cho DP, Cho TH. Placenta Therapy: Its Biological Role of AntiInflammation and Regeneration. In Placenta: chapter 8(113-134).2018. doi: 10.5772/intechopen.79718.

20. Bhansali S, Kumar V, Saikia U, Medhi B, Jha V, Bhansali A, et al. Effect of mesenchymal stem cells transplantation on glycaemic profile and their localization in streptozotocin induced diabetic Wistar rats. Indian J Med Res. 2015; 142(1): 63 71. doi:10.4103/0971-5916.162116.

21. Abdel Aziz MT, Atta HM, Mahfouz S, Fouad HH, Roshdy NK. Therapeutic potential of bone marrow-derived mesenchymal stem cells on experimental liver fibrosis. Clin Biochem. 2007; (40): 893-899. doi:10.1016/j.clinbiochem.2007.04.017.

22. Abdelhady A A, Diab M, Nabeeh A. Histological and biochemical alterations in the livers of rats treated with MSCs and placental extract against Doxorubicin as chemotherapy. Eur. J. Anat.2021; 25 (3): 325-338.

23. Graham ML, Janecek JL, Kittredge JA, Hering BJ, Schuurman HJ. The streptozotocin-induced diabetic nude mouse model: differences between animals from different sources. Comparative medicine. 2011; 61(4): 356-360.

24. Mohamed TA, Abouel-Nour MF, Eldemerdash R, Ibrahim Elgalady DAI. Therapeutic effects of bone marrow stem cells in diabetic rats. J. Comput. Sci. Syst. Bio. 2016; 9.

25. Bergmeyer H U, Horder M, Rey J. Approved recommendation on IFCC methods for the measurement of catalytic enzymes. Part 2: IFCC method for aspartate aminotransferase. J. Clin. Chem. Clin. Biochem. 1986; 24:497-510.

26. Mohamed J, Nafizah A N, Zariyantey A $\mathrm{H}$, Budin S. Mechanisms of diabetesinduced liver damage: the role of oxidative stress and inflammation. Sultan Qaboos University Medical Journal.2016; 16(2): e132.

27. Harris EH. Elevated liver function tests in type 2 diabetes. Clinical diabetes. 2005; 23(3): 115-119.

28. Rodríguez V, Plavnik L, de Talamoni NT. Naringin attenuates liver damage in streptozotocin-induced diabetic rats. Biomedicine \& Pharmacotherapy. 2018; 105, 95-102. doi: 10.1016/j.biopha.2018.05.120.

29. Salimuddin, Upadhyaya KC, Baquer NZ. Effects of vanadate on expression of liver araginase in experimental diabetic rats. IUBMB Life. 2008; 48(2), 237-40. doi: 10.1080/713803490.

30. Al-Ani IM, Al-Mishadani NS, Muslih RK, Hamoodi SR. Histological liver changes in streptozotocin induced diabetic mice. The International Medical Journal of Malaysia. 2009; 8(1).

31. Khalil MR, El-Demerdash RS, Elminshawy HH, Mehanna ET, Mesbah NM, Abo-Elmatty DM. Therapeutic effect of bone marrow mesenchymal stem cells in a rat model of carbon tetrachloride induced liver fibrosis. Biomedical journal. 2020; S2319-4170(20):30048-2. doi: 10.1016/j.bj.2020.04.011.

32. Zheng S, Yang J, Yang J, Tang Y, Shao Q, Guo L, et al. Transplantation of umbilical cord mesenchymal stem cells via different routes in rats with acute liver failure. Int J Clin Exp Pathol. 2015; 8:15854-15862.

33. Huang B, Cheng X, Wang H, Huang W, la Ga HZ, Wang D, et al. Mesenchymal stem cells and their secreted molecules predominantly ameliorate fulminant hepatic failure and chronic liver fibrosis in mice respectively. J Transl Med. 2016; 14:45. doi: 10.1186/s12967-016-0792-1.

34. Gilsanz C, Aller MA, Fuentes-Julian S, Prieto I, Blazquez-Martinez A, Argudo S, et al. Adipose-derived mesenchymal stem cells slow disease progression of acuteon-chronic liver failure. Biomed Pharmacother. 2017; 91:776-787. doi: 10.1016/j.biopha.2017.04.117.

35. Zhang Y. Li Y, Li W, Cai J, Yue M, Jiang $\mathrm{L}$, et al. Therapeutic effect of human umbilical cord mesenchymal stem cells at 
various passages on acute liver failure in rats. Stem cells international. 2018; 2018. doi: 10.1155/2018/7159465.

36. Wang YH, Wu DB, Chen B, Chen EQ, Tang H. Progress in mesenchymal stem cell-based therapy for acute liver failure. Stem cell research \& therapy. 2018; 9(1): 1-9. doi: 10.1186/s13287-018-0972-4.

37. Naughton GK. Regenerative Medicine Approaches for Engineering a Human Hair Follicle. In Principles of Regenerative Medicine. Academic Press. 2019; pp. 1297-1308.

38. Yamauchi A, Kamiyoshi A, Sakurai T, Miyazaki H, Hirano E, Lim HS, et al. Placental extract suppresses cardiac hypertrophy and fibrosis in an angiotensin II-induced cachexia model in mice. Heliyon. 2019; 5(10): e02655. doi: 10.1016/j.heliyon.2019.e02655.

39. Landek-Salgado MA, Rose NR, Caturegli P. Placenta suppresses experimental autoimmune hypophysitis through soluble TNF receptor 1 . Journal of autoimmunity. 2012; 38(2-3):J88-J96. doi:10.1016/j.jaut.2011.07.001.

40. Albus. Udo. Guide for the Care and Use of Laboratory Animals (8th ed.). 2012; 267-268. 